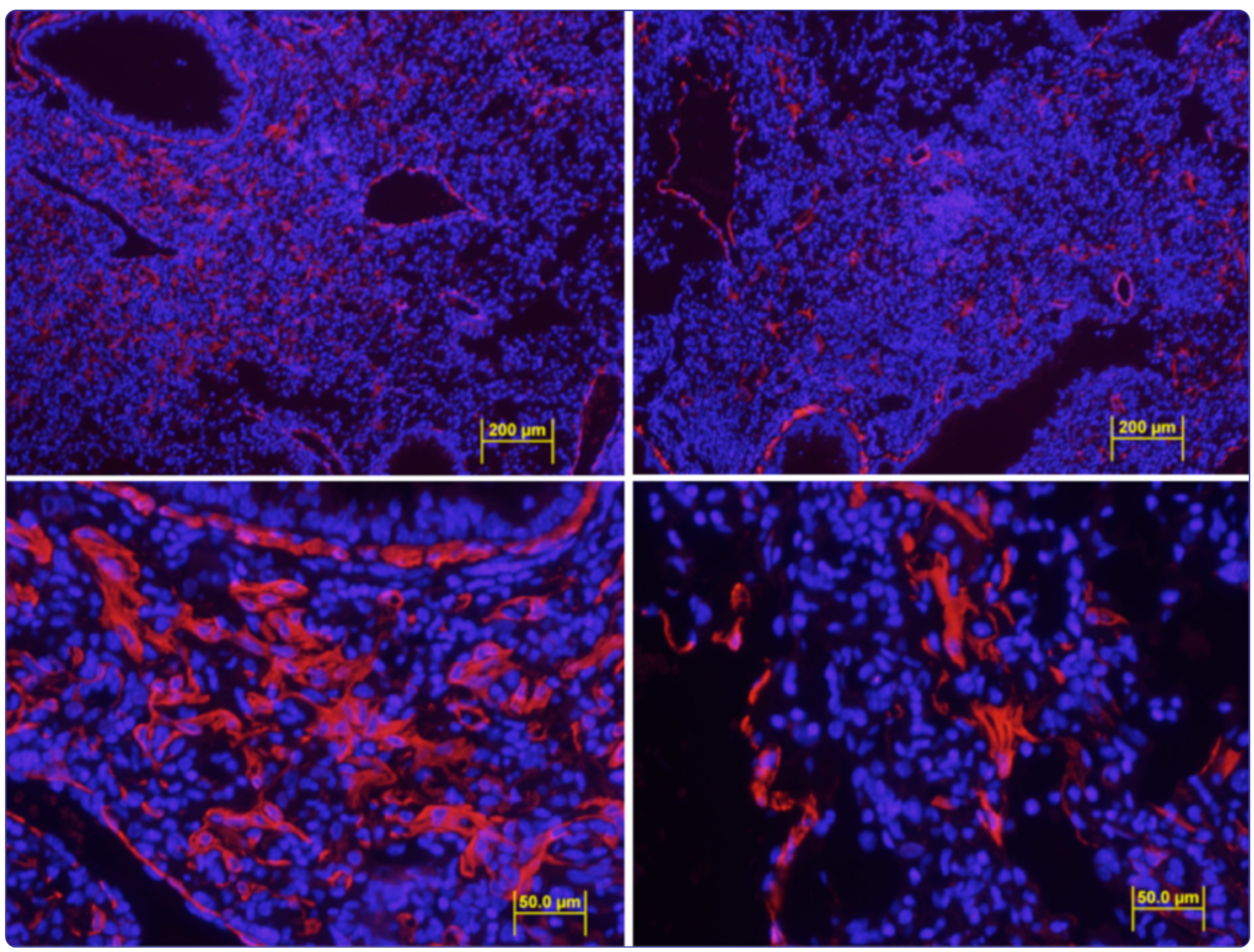

\title{
Role of IGF-1 pathway in lung fibroblast activation
}

Hung et al. 


\title{
Role of IGF-1 pathway in lung fibroblast activation
}

\author{
Chi F Hung, Maryam G Rohani, Sung-soon Lee, Peter Chen and Lynn M Schnapp*
}

\begin{abstract}
Background: IGF-1 is elevated in pulmonary fibrosis and acute lung injury, where fibroblast activation is a prominent feature. We previously demonstrated that blockade of IGF pathway in murine model of lung fibrosis improved outcome and decreased fibrosis. We now expand that study to examine effects of IGF pathway on lung fibroblast behaviors that could contribute to fibrosis.

Methods: We first examined mice that express aSMA promoter upstream of GFP reporter treated with A12, a blocking antibody to IGF-1 receptor, after bleomycin induced lung injury. We then examined the effect of IGF-1 alone, or in combination with the pro-fibrotic cytokine TGF $\beta$ on expression of markers of myofibroblast activation in vitro, including aSMA, collagen a1, type 1, collagen a1, type III, and TGF $\beta$ expression.

Results: After bleomycin injury, we found decreased number of aSMA-GFP + cells in A12 treated mice, validated by aSMA immunofluorescent staining. We found that IGF-1, alone or in combination with TGF- $\beta$, did not affect aSMA RNA expression, promoter activity, or protein levels when fibroblasts were cultured on stiff substrate. IGF-1 stimulated Colla1 and Col3a1 expression on stiff substrate. In contrast, IGF-1 treatment on soft substrate resulted in upregulation of aSMA gene and protein expression, as well as Col1a1 and Col3a1 transcripts. In conclusion, IGF-1 stimulates differentiation of fibroblasts into a myofibroblast phenotype in a soft matrix environment and has a modest effect on aSMA stress fiber organization in mouse lung fibroblasts.
\end{abstract}

Keywords: Fibroblasts, IGF, Fibrosis

\section{Introduction}

Insulin-like growth factor-1 (IGF-1) plays an important role in the development and homeostasis of many organs. IGF-1 acts as an important survival factor for various cells by inhibiting apoptosis and inducing cellular proliferation [1-3]. However, IGF-1 has also been implicated in disease states where pathologic fibrosis is the predominant feature. For example, in patients with systemic sclerosis (SSc), serum IGF-1 level is elevated in those with more severe skin involvement and pulmonary fibrosis [4]. Moreover, affected skin from subjects with SSc show 1.9 fold higher levels of IGF-1 mRNA expression compared to normal controls [4]. In the murine bleomycin lung injury model, IGF-1 mRNA was increased three to four fold over control in pulmonary fibrosis [5]. IGF-1 immunostaining was increased in lung tissues from patients with fibroproliferative

\footnotetext{
* Correspondence: Ischnapp@uw.edu

Center for Lung Biology, Division of Pulmonary and Critical Care Medicine, Department of Medicine, University of Washington, 850 Republican St, Box358052, Seattle 98109, WA, USA
}

ARDS [6] and IGF-1 levels were elevated in the bronchoalveolar lavage fluid (BALF) of patients with early ARDS [7]. We showed that IGF-1 provided a pro-survival signal to lung fibroblasts but not epithelial cells [8]. We further showed that blockade of the IGF-1 pathway in the murine bleomycin lung injury model hastened resolution of pulmonary fibrosis and increased fibroblast apoptosis [8]. In this study, we ask whether IGF-1 activates the myofibroblast phenotype. In addition to its role in cell survival, IGF-1 can alter gene expression and lead to phenotypic changes in fibroblasts [9-12]. The myofibroblast phenotype confers a number of important functional changes that play an important role in lung injury and repair $[13,14]$. We hypothesize that the IGF-1 pathway increases fibrosis in lung injury by activating fibroblasts to the $\alpha \mathrm{SMA}$-expressing myofibroblast phenotype. 


\section{Materials and methods Cells and reagents}

Recombinant IGF-1 and TGF- $\beta 1$ were purchased from R\&D Systems (Minneapolis, MN). Function-blocking antibody to the human IGF-1 receptor (A12) and keyhole limpet hemocyanin $(\mathrm{KLH})$ isotype control antibody were a generous gift from Dale Ludwig (ImClone Systems) $[15,16]$. A12 inhibits type 1 IGF receptor signaling in murine and human tissues and does not cross-react with the insulin receptor [15]. We verified that our preparation of A12 was endotoxin-free by Limulus Amebocyte Lysate assay (Cambrex BioScience). For detection of $\alpha \mathrm{SMA}$ by Western blot, antibody to $\alpha$ SMA (mouse IgG) was purchased from Sigma-Aldrich (clone 1A4). Horseradish peroxidase-conjugated anti-mouse IgG were purchased from Zymed (San Francisco, CA).

\section{Bleomycin-induced lung injury}

Animal protocol was approved by University of Washington Institutional Animal Care and Use Committee. Transgenic mice that express $\alpha$ SMA promoter upstream of GFP reporter construct on a C57Bl6 background ( $\alpha$ SMA-GFP mice) were a generous gift from Dr. Jen-Yue Tsai (National Eye Institute, NIH) [17]. Mice underwent intratracheal bleomycin instillation $(0.032 \mathrm{U} /$ mouse, SICOR Pharmaceuticals, Inc., Irvine, CA) as previously described [8]. Mice ( $n=4$ /group) received injections of either A12 $(40 \mathrm{mg} / \mathrm{kg}$ ) or KLH isotype control antibody intraperitoneally on $\mathrm{d} 7$ following bleomycin instillation and then twice weekly. The mice were sacrificed 21 days after bleomycin instillation. The right lungs were inflated to $25 \mathrm{~cm} \mathrm{H} 2 \mathrm{O}$ pressure, fixed with paraformaldehyde and paraffin embedded. $5 \mu \mathrm{m}$ thick sections were deparaffinized, rehydrated. For quantification of GFP $(+)$ cells, right middle lobe sections were systematically scanned in a microscope using 10x objective. Total number of GFP positive cells and DAPI positive cells were quantified in each successive field (NIH Image), v1.410). The mean score of all the fields was used for each mouse. For quantification of $\alpha$ SMA (+) cells, rehydrated right lung sections underwent heat antigen retrieval in buffer (Dako Target Retrieval Solution), incubated in blocking solution overnight at $4 \mathrm{C}$, and then immunostained with Cy3-conjugated anti- $\alpha$ SMA antibody (Sigma-Aldrich clone 1A4, 1:200) for $1 \mathrm{hr}$ at room temperature. The sections were counterstained with DAPI and mounted for visualization (Invitrogen Prolong Gold). Five predetermined fields were examined on each slide with $10 \times$ objective. The degree of $\alpha$ SMA staining was expressed as the ratio of red $\mathrm{Cy} 3$ staining area to DAPI staining area (NIH ImageJ, v1.41o). Airway and vascular-associated $\alpha$ SMA were masked in the image analysis. For confocal microscopy, bleomycin-injured mouse lungs from $\alpha$ SMA-GFP mice were inflated with $4 \%$ paraformaldehyde and fixed for 2 hours, submerged in
$18 \%$ sucrose at $4 \mathrm{C}$ overnight, and embedded and frozen in OCT. Frozen sections were immunostained for $\alpha \mathrm{SMA}$ (Cy3-conjugated anti- $\alpha$ SMA, Sigma-Aldrich clone 1A4, 1:200) and visualized under confocal microscopy.

\section{In vitro IGF-1 studies}

Mouse lung fibroblasts (MLF) isolated from C57/Bl6 or aSMA-GFP mice were maintained in DMEM with $10 \%$ FBS, $100 \mathrm{U} / \mathrm{ml}$ penicillin, $100 \mathrm{U} / \mathrm{ml}$ streptomycin and $5 \mathrm{mM}$ glutamate at $37^{\circ} \mathrm{C}$ in $5 \% \mathrm{CO}_{2}$ as previously described [18]. For some studies, MLF were isolated from C57/Bl6 mice three days after intratracheal instillation with saline (control) or bleomycin ( $\mathrm{n}=3$ mice/group). Unless otherwise indicated, experiments used MLF from C57/Bl6 wildtype mice. Cells were used between passages 2-5. MLF were grown to subconfluence and then plated either in 6-well tissue culture plates (Falcon) or 6well tissue culture plates coated with collagen matrix $(1 \mathrm{mg} / \mathrm{ml})$. To test the effect of a soft extracellular matrix on fibroblast response to IGF-1, we employed a collagen I gel matrix at a final concentration of $1 \mathrm{mg} / \mathrm{ml}$, which has been previously described to have an elastic modulus of $<100 \mathrm{~Pa}$ [19]. We mixed Collagen I $(3 \mathrm{mg} / \mathrm{ml})$ (BD Biosciences), MCDB (2X), and DMEM (with or without resuspended MLF) in 1:1:1 ratio. Immediately following mixing, the $\mathrm{pH}$ of the mixture was adjusted to neutral using $1 \mathrm{M} \mathrm{NaOH}$. The mixture was allowed to gelatinize at room temperature for 1 hour.

Following attachment, cells were serum-starved overnight and treated with IGF-1 $(100 \mathrm{ng} / \mathrm{ml})$, TGF- $\beta 1$ $(10 \mathrm{ng} / \mathrm{ml}$ or $1 \mathrm{ng} / \mathrm{ml})$, or IGF-1 $(100 \mathrm{ng} / \mathrm{ml}) /$ TGF- $\beta 1$ $(10 \mathrm{ng} / \mathrm{ml})$ for $24 \mathrm{hr}$, with the presence of A12 $(40 \mu \mathrm{g} / \mathrm{ml})$ or PI3 kinase inhibitor LY294002 (Calbiochem, $50 \mu \mathrm{M}$ ) in some experiments. Controls were serum-free media alone, and with A12 or LY294002 in experiments where the inhibitors were used. Parallel cultures were used for immunofluorescence studies, protein analysis, RNA analysis and promoter activity. All experiments were performed in triplicate, and repeated at least 3 times.

\section{Real-time PCR}

Total RNA was isolated from MLF using Qiagen RNeasy Mini Kit per manufacturer's specifications after treatment with the indicated growth factors. RNA quality was verified using Agilent Bioanalyzer. Total RNA was reverse-transcribed to cDNA using Applied Biosystems High-Capacity cDNA Archive Kit. Realtime PCR was done using ABI7900HT with the use of pre-designed primer and probes (ABI TaqMan Gene Expression Assays) for Hprt (Mm00446968_m1), and Acta2 (Mm01546133_m1), Col1a1 (Mm00801666_g1), Col3a1 (Mm01254476_m1), and Tgfb1 (Mm00441724_m1). Analysis was done using MS Excel calculating RQ by 2-DDCT. 


\section{aSMA promoter activity}

MLF isolated from $\alpha$ SMA-GFP mice were washed with PBS, trypsinized and fixed in paraformaldehyde. Flow cytometry (3000 cells per treatment group) was performed using the Guava PCA System (Guava Technologies, Hayward, CA) with the Guava ExpressPlus program and data analyzed using CellQuest 2.0 (BD Biosciences).

\section{Western blot analysis}

To assess $\alpha$ SMA protein expression, cells were washed in PBS and lysed in buffer containing $100 \mathrm{mM}$ Tri- $\mathrm{HCl}$ (pH 7.4), $150 \mathrm{mM} \mathrm{NaCl}, 1 \mathrm{mM} \mathrm{CaCl}_{2}, 0.1 \%$ SDS, $1 \%$ Triton-X, $0.1 \%$ NP-40, and protease inhibitor cocktail tablet (Roche). Protein concentrations were determined by the BCA assay (Pierce). Equal amounts of protein were separated by sodium dodecyl sulfate-polyacrylamide gel electrophoresis (SDS-PAGE), and electrophoretically transferred to PVDF membrane. Membranes were blocked with $5 \%$ nonfat dry milk/0.05\% Tween-20/PBS for $1 \mathrm{hr}$ at room temperature, incubated with mouse anti- $\alpha$ SMA IgG (1:20,000), rabbit anti-Collagen I IgG (Gene'Tex, 1:5,000), or rabbit anti-Collagen III IgG (Rockland, 1:5,000) overnight at $4^{\circ} \mathrm{C}$, washed with $0.1 \%$ Tween-20/PBS, incubated with horseradish peroxidase-conjugated goat anti-mouse IgG $(1: 10,000)$ for $1 \mathrm{hr}$, washed with $0.1 \%$ Tween-20/PBS and then developed with enhanced chemiluminescence (ECL) technique (Amersham, England). Densitometric analysis of relative band intensities was performed by analyzing scanned blots with NIH Image J (version 1.41o). Values are normalized to GAPDH control and presented as relative intensities compared to control (serum-free condition).

\section{aSMA and filamentous actin (F-actin) Co-staining}

To assess $\alpha$ SMA fiber organization, primary MLF at P1 were treated with IGF-1 (100 ng/ml), TGF- $\beta 1$ (10 ng/ml) or IGF-1/TGF- $\beta 1$ (100 ng/ml and $10 \mathrm{ng} / \mathrm{ml}$, respectively) for $24 \mathrm{hr}$, then fixed with $4 \%$ paraformaldehyde at RT $\times 10$ min followed by permeabilization with $0.5 \%$ Triton-X100 in PBS at RT $\times 3$ min. The fixed cells were blocked in $1 \%$ $\mathrm{BSA}$ in PBS $\times 20 \mathrm{~min}$ at RT and incubated with primary antibody to $\alpha$ SMA (Abcam, rabbit IgG) overnight at $4^{\circ} \mathrm{C}$, then incubated with Alexa488-conjugated secondary antibody (Invitrogen, goat anti-rabbit IgG), followed by incubation with Alexa 564-conjugated phalloidin (5 units/ml, Invitrogen) at RT for $20 \mathrm{~min}$ for F-actin staining. Nuclei were counterstained with DAPI. Ten to twelve random fields $(20 \times)$ per treatment condition were analyzed for cells staining for $\alpha$ SMA stress fibers (green) and F-actin fibers (red). The total number of F-actin staining cells per field was counted. Of the F-actin + cells counted, the number of $\alpha$ SMA stress fiber + cells were counted. Results are presented as percentage of $\alpha$ SMA stress fiber + cells out of the total number of F-actin + cells. Imaging was performed with the assistance of the Lynn and Mike Garvey Cell Imaging Laboratory at the UW Institute for Stem Cell and Regenerative Medicine. Images were obtained using a Nikon TiE inverted widefield fluorescence microscope and analyzed by NIH ImageJ (version 1.41o).

\section{Statistical analysis}

Means of more than two groups of data were compared using one-way analysis of variance (ANOVA) for analysis of one independent variable or two way ANOVA, for analysis of two independent variables, followed by Tukey's honestly significant difference (HSD) post hoc test. Student T-test was used for comparison of parametric data. All tests were two-tailed and $p$ values $\leq 0.05$ were considered significant. Statistical analysis was performed using GraphPad Prism for Macintosh version 4.0c (GraphPad Software).

\section{Results}

\section{Blockade of IGF-1 pathway in vivo decreases aSMA} expression after injury

We previously demonstrated that IGF-1 is upregulated in patients with acute lung injury and in mice following bleomycin-induced lung injury [7,8]. Furthermore, IGF-1 receptor blockade hastened resolution of fibrosis in mouse model of injury [8]. To determine whether IGF-1 blockade attenuates myofibroblast activation, we examined $\alpha$ SMA-GFP transgenic mice after bleomycin-induced lung injury with or without A12 antibody (IGF-1R antibody) treatment. Representative H\&E images from bleomycin injured $\alpha$ SMA-GFP transgenic mice show decreased fibrotic regions in the peribronchiolar regions following bleomycin injury (Figure 1A). $\alpha \mathrm{SMA}$ immunostaining shows colocalization of $\alpha$ SMA with GFP expression, demonstrating GFP expression as a reliable surrogate for $\alpha$ SMA expression following bleomycin injury in this transgenic model (Figure 1B). Following bleomycin-induced lung injury, we found significantly fewer GFP positive cells (as a marker of $\alpha$ SMA expression) (Figure $1 C$ ) in the A12 treated group. Interestingly, the most striking difference visually between the two groups was in areas of relatively normal architecture $(a, b)$. To validate our findings in GFP, we immunostained lung sections for $\alpha$ SMA. We also found decreased $\alpha$ SMA expression in bleomycin-injured lungs that were treated with A12, consistent with our GFP findings (Figure 1D). In uninjured mice treated with A12 alone, there was no difference in GFP expression (not shown).

\section{Effect of IGF-1 treatment on aSMA promoter activity}

In addition to an effect on cell survival, another potential explanation for decreased GFP $(\alpha \mathrm{SMA}+)$ cells is a direct effect of IGF-1 blockade on fibroblast $\alpha$ SMA 


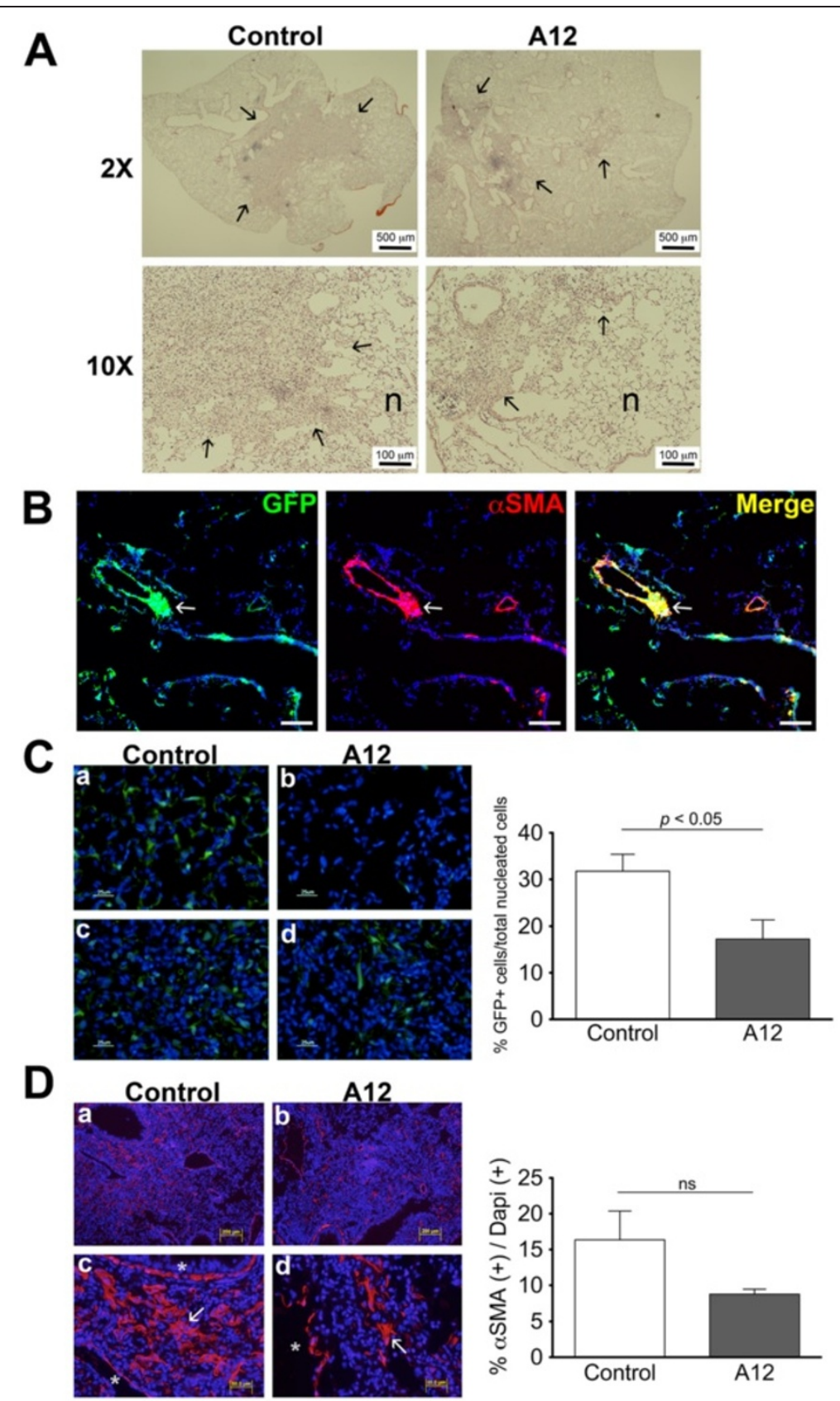

Figure 1 Decreased aSMA promoter activity and aSMA protein expression in bleomycin-injured mice treated with IGF-1 receptor blocking antibody (A12). (A) Representative H\&E sections of aSMA-GFP mice at day 21 after bleomycin injury. Fibrotic regions of lung parenchyma are indicated by $(\mathbf{N})$ and normal lung parenchyma are indicated by (n). (B) aSMA immunostaining of bleomycin-injured lung in an aSMA-GFP mouse. Note the overlap of aSMA staining (red) with aSMA-GFP expression (green) in the peribronchiolar fibrotic region indicated by $(\mathbf{N})$. Scale bars represent $100 \mu \mathrm{m}$. (C) (Left) Representative fluorescent images of aSMA-GFP mice treated with A12 (b and d) showed less aSMA promoter activity as indicated by GFP (green) positive cells, compared to control mice (a and $\mathbf{c}$ ) at d21 after bleomycin instillation. (Right) Percentage of aSMA-GFP + cells/total number of DAPI + cells, quantification by NIH ImageJ ( $n=4$ mice/group, mean \pm SEM). (D) (Left) Representative images of aSMA staining by immunofluorescent microscopy of the same A12-treated mice (b and $\mathbf{d}$ ) compared to control mice ( $\mathbf{a}$ and $\mathbf{c}$ ) at d21 after bleomycin instillation. Large airways and vasculature staining for aSMA, indicated by an asterisk $\left(^{*}\right.$ ), were masked in the analysis. Interstitial staining, indicated by an arrow $(\mathbf{N})$, was included in the analysis. (Right) Ratio of aSMA staining area per DAPI + area ( $n=4$ mice/group, mean \pm SEM). 
expression. Therefore, we asked whether IGF-1 affected fibroblast $\alpha$ SMA expression alone or synergistically with pro-fibrotic cytokine TGF- $\beta 1$, and whether this effect can be blocked by treatment with the IGF-1 receptorblocking antibody A12.

First, we evaluated $\alpha$ SMA promoter activity (measured by mean GFP intensity) in MLF isolated from $\alpha$ SMAGFP mice. TGF- $\beta 1$ but not IGF- 1 increased $\alpha$ SMA promoter activity, and no synergistic effect was seen with IGF-1 and TGF- $\beta 1$ co-stimulation (Figure 2). These results suggest IGF-1 does not regulate $\alpha$ SMA promoter activity in MLF either alone, or synergistically with TGF$\beta 1$, a cytokine known to upregulate $\alpha$ SMA RNA expression in the conditions tested.

\section{Effect of IGF-1 treatment on transcription of myofibroblast markers}

Next, we assessed the role of IGF-1 on $\alpha$ SMA mRNA expression by real-time PCR. MLF grown on tissueculture plate or collagen I-coated tissue culture plates (stiff substrates) were treated with IGF-1, TGF- $\beta 1$ or combination of IGF- 1 and TGF- $\beta 1$ to assess for synergy. As expected, treatment with positive control TGF- $\beta 1$ increased Acta2 expression (Figure 3A). However, IGF-1 did not increase Acta2 expression. Co-incubation of IGF- 1 and TGF- $\beta 1$ did not alter the TGF- $\beta 1$ induced
Acta2 expression. Finally, treatment of cells with A12 did not change Acta 2 expression in any of the conditions, including TGF- $\beta 1$ treatment (Additional file 1: Figure S1). We further confirmed that MLF isolated from bleomycin-injured mice did not respond differently to cytokine stimulation than MLF from uninjured mice: MLF isolated from bleomycin-injured mice and treated with IGF-1 did not increase $\alpha$ SMA RNA expression (Figure 3C).

aSMA expression is one of several changes observed with fibroblast activation. Activated fibroblasts may also increase TGF- $\beta 1$ expression and synthesis of extracellular matrix proteins such as collagen $\alpha 1$, type I and collagen $\alpha 1$, type III. Therefore, we measured transcriptional changes in these genes after treatment with IGF-1, TGF$\beta 1$, or both. As expected, treatment with TGF- $\beta 1$ increased Col1a1 expression. Interestingly, IGF-1 treatment led to a 1.5 fold increase in Colla1 expression over serum-free control at 24 hours (Figure 3A) and the effect was inhibited by A12 (Additional file 1: Figure S1). IGF-1 treatment also led to a 1.5 fold increase in Col3a1 expression.

The effect of IGF-1 treatment on MLF is not mediated through interaction with TGF- $\beta 1$. No significant synergy was seen between IGF- 1 and TGF- $\beta 1$, and IGF- 1 blockade with A12 did not affect TGF- $\beta 1$ stimulated MLF
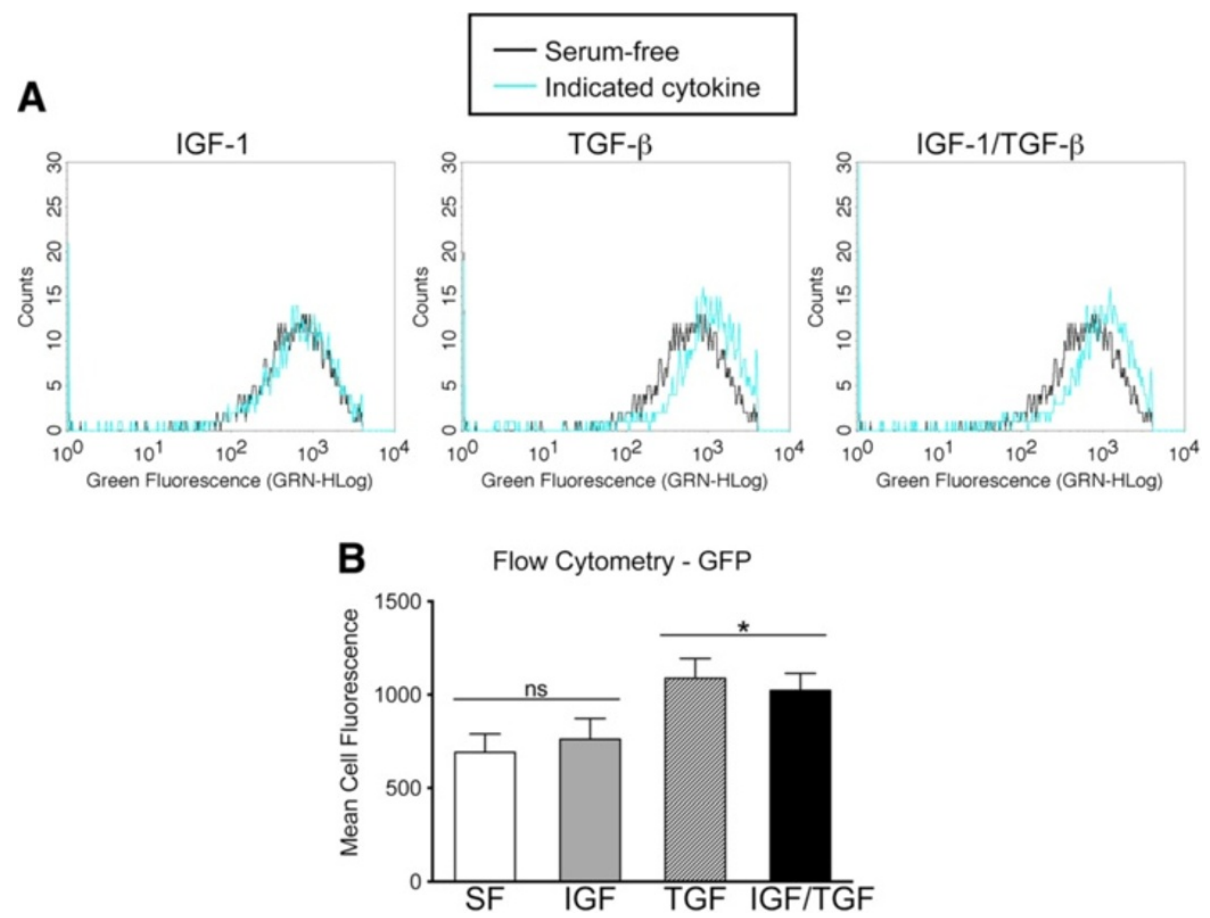

Figure 2 Effect of IGF-1 on aSMA promoter activity in aSMA-GFP transgenic mice. (A) MLF from aSMA-GFP mice were treated with IGF-1 $(100 \mathrm{ng} / \mathrm{ml})$, TGF $\beta(10 \mathrm{ng} / \mathrm{ml})$, or both on tissue culture plate for $24 \mathrm{hr}$. Control group was maintained in serum-free media. Representative histogram of each treatment condition overlaying control group histogram is shown. (B) Bar graph summarizing the mean green fluorescence of each treatment condition. 3000 cells/condition analyzed. ${ }^{*} p<0.05$ compared to serum-free control. 
A

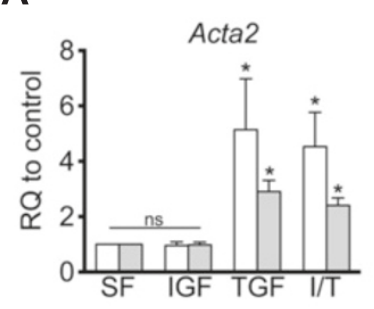

\section{B}

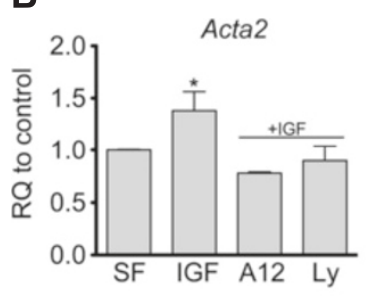

Stiff substrate
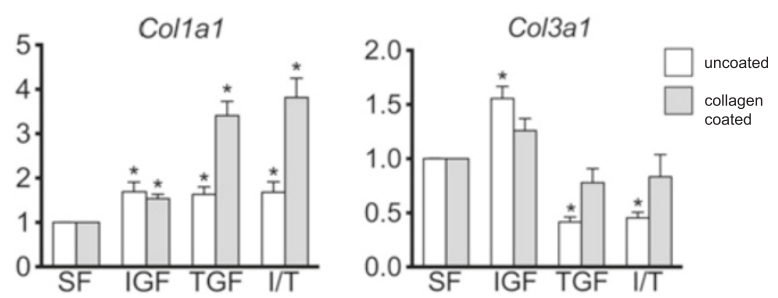

Soft substrate
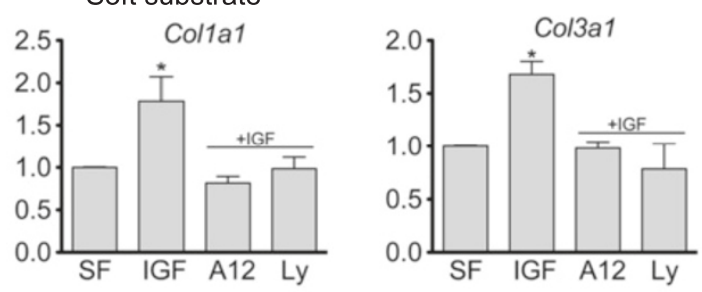

C

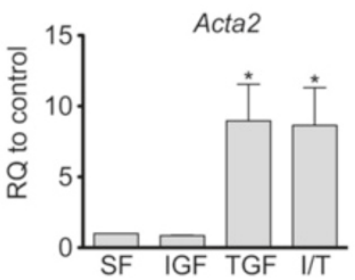

MLF from bleomycin injured lungs
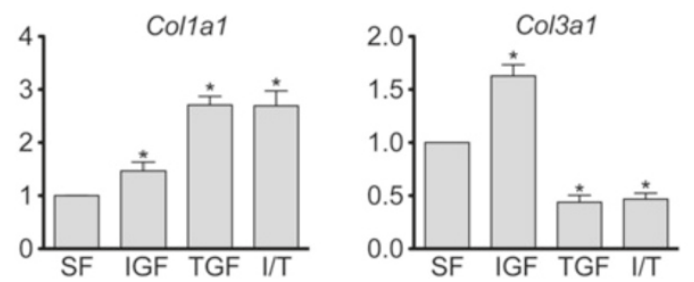

Figure 3 Effect of matrix stiffness on response to IGF-1 treatment. (A) MLF on tissue culture plate or collagen I-coated tissue culture plate (stiff substrates) were treated with IGF-1 $(100 \mathrm{ng} / \mathrm{ml})$, TGF $\beta(1 \mathrm{ng} / \mathrm{ml})$ or IGF/TGF $\beta(100 \mathrm{ng} / \mathrm{ml}$ and $1 \mathrm{ng} / \mathrm{ml}$, respectively), or serum-free media (negative control) for $24 \mathrm{hr}$. (B) MLF on collagen I (1 mg/ml) hydrogel (soft substrate) were treated with IGF-1 (100 ng/ml), IGF-1 $(100 \mathrm{ng} / \mathrm{ml})$ with A12 $(40 \mu \mathrm{g} / \mathrm{ml})$ or PI3 kinase inhibitor LY294002 (Ly, $50 \mu \mathrm{M})$ for $24 \mathrm{~h}$. (C) MLF isolated from bleomycin-injured C57Bl6 mice were treated with the indicated cytokine. Real time PCR analyses of myofibroblast markers Acta2, Col1a1, and Col3a1 were performed. Data were normalized to HPRT expression. Y-axis represents fold increase compared to serum-free control $\left(n=3\right.$, mean $\pm S E M,{ }^{*} p<0.05$ compared to serum-free control).

(Additional file 1: Figure S1). Moreover, IGF-1 treatment no effect on $T g f b 1$ expression in MLF (Additional file 2: Figure S2). Similarly, bleomycin did not affect MLF responsiveness to IGF-1 stimulation in vitro as similar results were obtained using MLF isolated from bleomycininjured lungs (Figure 3C).

\section{Effect of IGF-1 treatment on aSMA and matrix protein expression}

Similar to findings with RNA expression, IGF-1 treatment on stiff substrate did not increase $\alpha S M A$ protein expression in MLFs after $24 \mathrm{hr}$ (Figure 4A and C). TGF$\beta 1$ treatment on stiff substrate increased $\alpha$ SMA protein expression, but the addition of IGF-1 had no synergistic effect. Similarly, MLF isolated from bleomycin-injured mice did not show increased $\alpha$ SMA protein expression with IGF-1 treatment compared to MLF isolated from control mice (data not shown). In addition to $\alpha \mathrm{SMA}$, we also assessed in vitro expression of matrix proteins Col I and III. Consistent with our findings in Colla1 and Col3a1 transcriptional activity, MLF treated with IGF-1 demonstrated increased Col I and III expression (Figure 4B and C).

\section{Effect of IGF-1 on stress fiber formation}

The percentage of cells staining for F-actin fibers (as indicated by phalloidin staining) and the intensity of Factin fiber staining did not significantly change with IGF-1 treatment. However, IGF-1 treatment modestly increased the percentage of cells with $\alpha$ SMA-containing stress fibers compared to serum-free control (33\% vs $19.5 \%, \mathrm{p}=0.05$ ) (Figure 5). Furthermore, this increase was blocked by A12. As expected, TGF- $\beta 1$ treatment increased the percentage of $\alpha \mathrm{SMA}$ stress fiber positive fibroblasts and the intensity of phalloidin staining. However, there was no further increase in the percentage of fibroblasts with $\alpha$ SMA-containing stress fibers with the addition of IGF-1 to TGF $\beta$ treatment. A12 had no effect on TGF- $\beta 1$-mediated increase in $\alpha$ SMA-containing stress fibers (Figure 5). 


\section{Protein expression on stiff substrate}

A

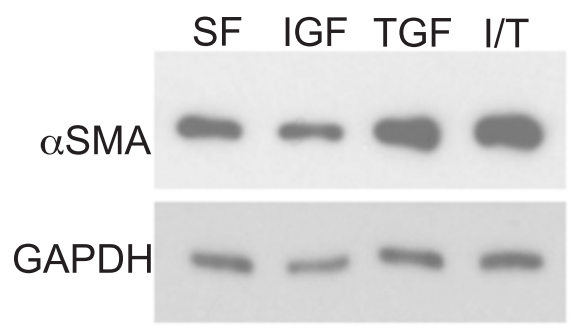

B
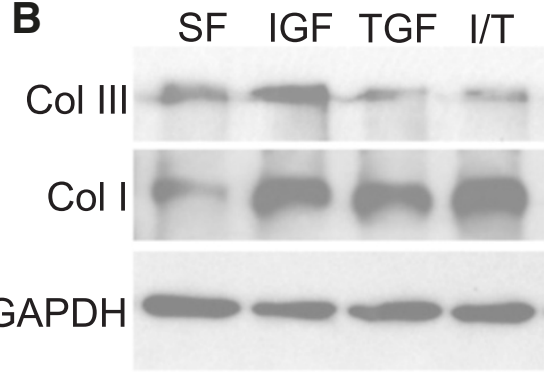

C
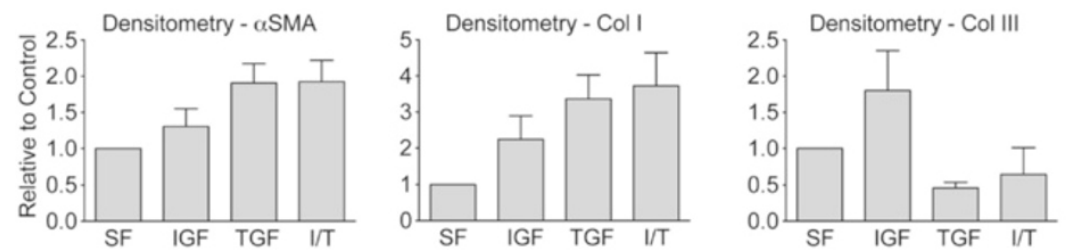

\section{$\alpha$ SMA expression on soft substrate}
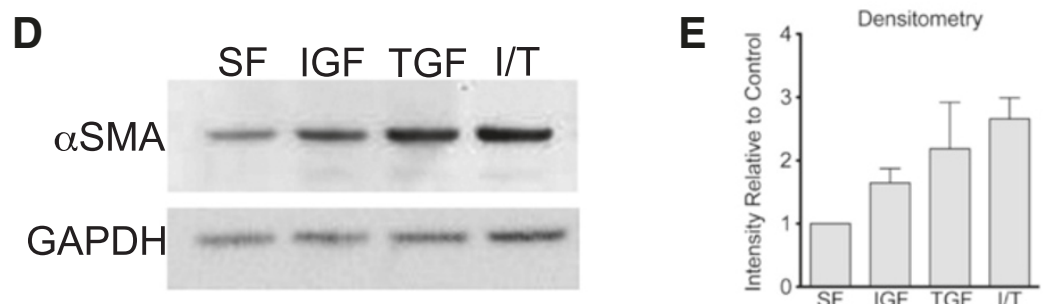

Figure 4 Effect of IGF-1 on protein expression in soft and stiff substrates. (A-B) MLF on tissue culture plate (stiff substrate) were stimulated with IGF-1, TGF- $\beta 1$, or IGF-1/TGF- $\beta 1$ for 24 hr. Representative Western blots of aSMA, Col I, and Col III with GAPDH loading control are shown. (C) Densitometry after normalization to loading control. (D) MLF cultured on soft matrix and treated with IGF, TGF- $\beta 1$, or IGF/ TGF- $\beta 1$ for 24 hr. Representative Western blots of aSMA with GAPDH loading control are shown. (E) Densitometry after normalization to loading control $(n=3$, mean \pm SEM).

Role of matrix stiffness in response to IGF stimulation Since MLF rapidly differentiate into myofibroblasts when grown on tissue culture plastic, we questioned whether the high level of baseline myofibroblast activation obscured the effects of IGF-1 on $\alpha$ SMA expression. Therefore, we cultured MLF on collagen I gel matrix (soft substrate) and asked whether IGF-1 increased myofibroblast activation in these conditions. In contrast to MLF grown on tissue culture plastic, MLF grown on soft substrate up-regulated Acta2 expression in response to IGF-1 (Figure 3B). Likewise, MLF grown on soft substrate significantly increased $\alpha$ SMA protein expression in response to IGF-1 (Figure 4D,E). IGF-1 treatment also increased Colla1 and Col3a1 expression on soft substrate (Figure 3B). Treatment with TGF- $\beta 1$, alone or in combination with IGF-1, resulted in a 2 -fold increase in $\alpha \mathrm{SMA}$ protein expression without synergistic effect with IGF-1 (Figure 4D,E). To ensure the soft biomechanical property of the collagen I gel substrate, rather than the presence of Collagen I in the substrate, was responsible for the effect of IGF-1 on $\alpha$ SMA, Col I and III, we also assessed the effect of IGF-1 on MLF cultured on collagen I-coated tissue culture plates. Similar to our findings in uncoated tissue culture plates, IGF-1 treatment had no effect on Acta2 expression and stimulated Colla1 and Col3a1 transcriptional activity (Figure 3A). Furthermore, the effect of IGF-1 on soft matrix was blocked by treatment with A12 blocking antibody (Figure 3B). We previously examined the signal transduction pathway activated by IGF-1 in MLF, and found that IGF-1 treatment led to phosphorylation of IRS-2 but not IRS-1, and phosphorylation of Akt [8]. The PI3-kinase pathwayis thus the likely pathway involved in IGF-1 signaling. When MLF grown on soft substrate was treated with PI3-kinase inhibitor Ly294002, the effect of IGF-1 on MLF was also blocked (Figure 3B). Together, these data suggest that the mechanical properties of the matrix modulate the response of MLF to IGF-1 stimulation. 


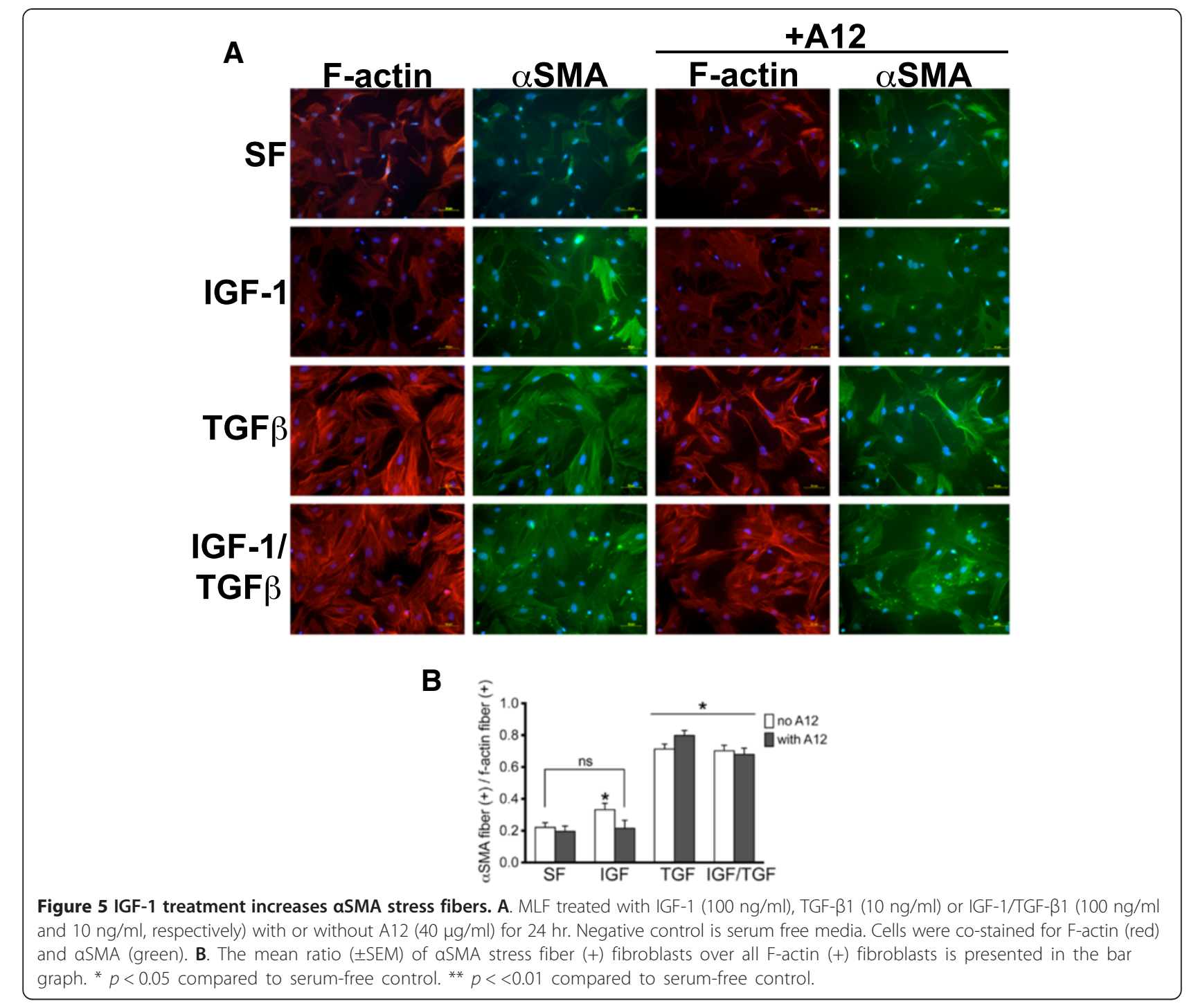

\section{Discussion}

IGF-1 has been reported to activate fibroblasts into the myofibroblast phenotype $[9,12,20-22]$. We previously demonstrated that IGF-1 provided an important prosurvival signal to fibroblasts in lung injury [8]. We now demonstrate in $\alpha$ SMA-GFP mice that IGF-1 pathway blockade decreases $\alpha \mathrm{SMA}$ + fibroblasts after bleomycin lung injury. In addition to an effect on cell survival, we asked whether IGF-1 induces fibroblast differentiation into myofibroblasts, which may also explain the observed decrease in GFP + and $\alpha \mathrm{SMA}+$ cells in our lung injury model with IGF-1 blockade.

Myofibroblasts are specialized fibroblasts that exhibit a contractile phenotype as a result of increased stress fiber formation, $\alpha$ SMA expression, development of mature focal adhesions, and enhanced extracellular matrix deposition [9,23-25]. The phenotypic transformation confers a number of important functional changes that play an important role in lung injury and repair $[13,14]$. Expression of $\alpha \mathrm{SMA}$, considered a hallmark of the myofibroblast phenotype, contributes to the contractile phenotype that plays an important role in fibrosis [26,27].

Previous studies on the effect of IGF-1 on myofibroblast differentiation and $\alpha \mathrm{SMA}$ expression have shown conflicting results. In one study, human fetal lung fibroblasts treated with IGF-1 increased $\alpha$ SMA and collagen I synthesis [11]. In another study, human colonic fibroblast cell lines treated with IGF-1 showed a small increase in $\alpha S M A$ expression that was significantly less than the upregulation induced by TGF- $\beta 1$ [12]. Similarly, IGF-1 treatment did not increase $\alpha$ SMA expression in primary human corneal fibroblasts [12,21]. Direct comparison of different studies is complicated by the fact that fibroblasts from different species, organs and stages of development respond differently to fibrogenic stimuli [28-30]. Moreover, recent studies show that extracellular 
biomechanical properties (i.e. matrix stiffness) regulate myofibroblast differentiation and modulate response to profibrotic cytokines such as TGF- $\beta 1$ [31-34]. Our data suggest IGF-1 is a profibrotic cytokine under soft extracellular matrix conditions, inducing expression of Acta2, Col1a1, and Col3a1. On stiff substrates, IGF-1 had no effect on $\alpha$ SMA gene or protein expression, alone or synergistically with TGF- $\beta 1$. On the other hand, the effect of IGF-1 on Col I and III gene and protein expression is maintained in both stiff and soft matrices. We previously demonstrated that IGF-1 stimulation of MLF induced IRS-2 and Akt phosphorylation, suggesting that IRS-2 and PI3 kinase are the major pathways activated by IGF-1 under the conditions tested [8]. In our present study, administration of PI3 kinase inhibitor blocked up-regulation of $\alpha$ SMA, Col I and III in IGF-1-treated MLFs, supporting our previous finding that PI3 kinase is an important downstream pathway in IGF-1-stimulated MLF.

We also investigated whether IGF-1 acted synergistically with TGF- $\beta 1$, a common profibrotic cytokine. In some studies, TGF- $\beta 1$ treatment of fibroblasts increased IGF-1 expression [12,21], raising the possibility of an autocrine effect of IGF-1 on $\alpha$ SMA expression. However, in our experiments, co-stimulation with IGF- 1 and TGF- $\beta 1$ did not enhance TGF- $\beta 1$-mediated $\alpha$ SMA expression, and A12 treatment did not block TGF- $\beta 1$-induced $\alpha$ SMA expression. Additionally, IGF- 1 treatment did not alter TGF- $\beta 1$ gene expression in MLF. Together, our data suggest the TGF- $\beta 1$-mediated changes are independent of the IGF-1 pathway.

An interesting finding in our present study is that IGF-1 exerts differential effects on MLF depending on the stiffness of the extracellular matrix. IGF-1 directly up-regulates Col I and III expression in both soft and hard matrices. This finding is consistent with our previously published in vivo findings where IGF-1 blockade led to decreased fibrosis as measured by hydroxyproline content at day 21 after bleomycin injury [8]. On the other hand, IGF-1 only regulates $\alpha$ SMA expression in soft matrix conditions. Liu and Tschumerplin previously demonstrated by atomic force microscopy that normal lung parenchyma constitute a soft matrix environment for fibroblasts whereas established fibrotic regions are significantly stiffer [35]. We previously demonstrated a significant increase in IGF-1 mRNA expression during early lung injury in mouse model [8] and increased IGF1 in bronchoalveolar lavage fluid in early ARDS [7]. We also found that IGF-1 receptor mRNA expression profile is similar to IGF-1 after bleomycin injury (unpublished). Together, these data implicate a temporal influence on the pro-fibrotic function of IGF-1. In early injury, prior to scar formation, IGF-1 may act as a pro-fibrotic cytokine on resident fibroblasts that reside in a compliant extracellular matrix, inducing $\alpha$ SMA expression and collagen deposition by fibroblasts. Later in injury, IGF-1 exerts anti-apoptotic effects on activated myofibroblasts and enhances collagen deposition. Thus, in our in vivo model, the decrease in $\alpha$ SMA-GFP + cells observed at day 21 with IGF-1 blockade may be due to decreased myofibroblast differentiation during the early phase of injury and increased apoptosis of fibrogenic myofibroblasts during the resolution phase.

We also found an increase in percentage of fibroblasts exhibiting $\alpha$ SMA-containing stress fibers. These results suggest that IGF-1 promotes the assembly of pre-formed aSMA units into filamentous form (stress fibers), rather than inducing expression of $\alpha$ SMA. Currently, the only demonstrated stimulus for the recruitment of cytosolic aSMA units into stress fibers is mechanical tension mediated by the formation of super-mature focal adhesions [36]. As previously shown, incorporation of $\alpha$ SMA into stress fibers enhances the contractility of myofibroblasts [27], which contributes to the restrictive phenotype seen in fibrotic lung diseases.

Our study has several important limitations. Fibroblasts cultured on stiff substrates such as tissue culture plates invariably become activated, potentially masking a true effect IGF-1 may have on myofibroblast differentiation. Evaluation of the IGF-1 pathway on substrates that mimic the physiologic stiffness of fibrotic lung $(\sim 20 \mathrm{kPa}$ Young's modulus) will be needed to fully assess whether IGF-1 also directly induces the myofibroblast phenotype in stiff lung matrix [31,33,35,37]. Additionally, evaluation of the IGF-1 pathway in vitro isolates fibroblasts from their native extracellular matrix and surrounding cellular environment. Cell-matrix interactions and non-fibroblast cellular mediators of myofibroblast differentiation where IGF-1 may also exert its profibrotic effect are absent in our in vitro studies. While the presence of collagen I in the growth substrate did not affect MLF responsiveness to IGF-1 treatment in our studies, we cannot exclude the possibility that the observed effects in collagen I gels were due to the three-dimensional substrate versus a twodimensional substrate.

In summary, IGF-1 blockade decreased myofibroblasts after bleomycin lung injury in $\alpha$ SMA-GFP reporter mice. IGF-1 plays a complex role in lung myofibroblast activation. IGF-1 regulates $\alpha$ SMA expression only in soft substrates while it enhances expression of other myofibroblast markers such as Col1a1 and Col3a1 in soft and stiff substrates. We conclude that IGF-1 stimulates myofibroblast differentiation by activating $\alpha$ SMA expression and matrix synthesis. Furthermore, the role of IGF-1 in fibroblast activation is dependent on the biomechanical properties of the extracellular matrix. Our present study highlights the complex biology of fibrosis where the pro-fibrotic effects of different growth factors are 
dependent on the time course of injury and repair as well as the biomechanical properties of the extracellular matrix.

\section{Additional files}

Additional file 1: Figure S1. Effect of A12 on cytokine treatment in stiff substrate. MLF on tissue culture plate were stimulated with IGF-1, TGF- $\beta 1$ ( $10 \mathrm{ng} / \mathrm{ml})$, or IGF-1/ TGF- $\beta 1$ for 24 or $48 \mathrm{hr}$ with or without A12 $(40 \mu \mathrm{g} / \mathrm{ml})$. Negative control is serum free media. Real time PCR analysis of myofibroblast markers Acta2 and Colla1 was performed. Data were normalized to HPRT expression. Y-axis represents fold increase compared to serum-free control $\left(n=3,{ }^{*} p<0.05\right.$ compared to serum-free control, **p $<0.05$ compared to no A12, \# not significant compared to no A12).

Additional file 2: Figure S2. Treatment with IGF-1 did not affect TgfbI gene expression. MLF on tissue culture plate, soft substrate, or from bleomycin-injured lungs were stimulated with IGF-1, TGF- $\beta 1$ (10 ng/ml), or IGF-1/TGF- $\beta 1$ for $24 \mathrm{hr}$. Negative control is serum free media. Real time PCR analysis of $T g f b 1$ was performed. Data were normalized to HPRT expression. Y-axis represents fold increase compared to serum-free control $(n=3$, mean \pm SEM).

\section{Competing interest}

LMS holds a patent entitled "Compositions And Methods For The Treatment Of Respiratory Disorders".

\section{Authors' contributions}

$\mathrm{CH}$ participated in study design, data acquisition and analysis and draft the manuscript, MR participated in data acquisition and analysis, SSL participated in data acquisition and analysis, LMS conceived of the study, and participated in its design and coordination and critically revised drafts of the manuscript. All authors read and approved the final manuscript.

\section{Acknowledgement}

This work was supported by American Heart Association Grant-in-Aid, NIH HL083481, and K24HL068796 (LMS). The imaging studies were supported in part by a gift to the Institute for Stem Cell and Regenerative Medicine. The authors thank Drs. Samuel Ash and Hoon Jung for technical assistance.

Received: 28 December 2012 Accepted: 4 October 2013

Published: 8 October 2013

\section{References}

1. Kurmasheva RT, Houghton PJ: IGF-I mediated survival pathways in normal and malignant cells. Biochim Biophys Acta 2006, 1766:1-22.

2. LeRoith $D$, Roberts $C T$ Jr: The insulin-like growth factor system and cancer. Cancer Lett 2003, 195:127-137.

3. Romano G: The complex biology of the receptor for the insulin-like growth factor-1. Drug News Perspect 2003, 16:525-531.

4. Hamaguchi Y, Fujimoto M, Matsushita T, Hasegawa M, Takehara K, Sato S: Elevated serum insulin-like growth factor (IGF-1) and IGF binding protein-3 levels in patients with systemic sclerosis: possible role in development of fibrosis. J Rheumatol 2008, 35:2363-2371.

5. Maeda A, Hiyama K, Yamakido H, Ishioka S, Yamakido M: Increased expression of platelet-derived growth factor $A$ and insulin-like growth factor-I in BAL cells during the development of bleomycin-induced pulmonary fibrosis in mice. Chest 1996, 109:780-786.

6. Krein PM, Sabatini PJ, Tinmouth W, Green FH, Winston BW: Localization of insulin-like growth factor-l in lung tissues of patients with fibroproliferative acute respiratory distress syndrome. Am J Respir Crit Care Med 2003, 167:83-90.

7. Schnapp LM, Donohoe S, Chen J, Sunde DA, Kelly PM, Ruzinski J, Martin T, Goodlett DR: Mining the acute respiratory distress syndrome proteome: identification of the insulin-like growth factor (IGF)/IGF-binding protein-3 pathway in acute lung injury. Am J Pathol 2006, 169:86-95.

8. Choi JE, Lee SS, Sunde DA, Huizar I, Haugk KL, Thannickal VJ, Vittal R, Plymate SR, Schnapp LM: Insulin-like growth factor-I receptor blockade improves outcome in mouse model of lung injury. Am J Respir Crit Care Med 2009, 179:212-219.
9. Goldstein RH, Poliks CF, Pilch PF, Smith BD, Fine A: Stimulation of collagen formation by insulin and insulin-like growth factor I in cultures of human lung fibroblasts. Endocrinology 1989, 124:964-970.

10. Scarpa RC, Carraway RE, Cochrane DE: Insulin-like growth factor (IGF) induced proliferation of human lung fibroblasts is enhanced by neurotensin. Peptides 2005, 26:2201-2210.

11. Chetty A, Cao GJ, Nielsen HC: Insulin-like Growth Factor-I signaling mechanisms, type I collagen and alpha smooth muscle actin in human fetal lung fibroblasts. Pediatr Res 2006, 60:389-394.

12. Simmons JG, Pucilowska JB, Keku TO, Lund PK: IGF-I and TGF-beta1 have distinct effects on phenotype and proliferation of intestinal fibroblasts. Am J Physiol Gastrointest Liver Physiol 2002, 283:G809-818.

13. Phan SH: The myofibroblast in pulmonary fibrosis. Chest 2002, 122:286S-2895.

14. Scotton CJ, Chambers RC: Molecular targets in pulmonary fibrosis: the myofibroblast in focus. Chest 2007, 132:1311-1321.

15. Burtrum D, Zhu Z, Lu D, Anderson DM, Prewett M, Pereira DS, Bassi R, Abdullah R, Hooper AT, Koo H, Jimenez X, Johnson D, Apblett R, Kussie P, Bohlen P, Witte L, Hicklin DJ, Ludwig DL: A fully human monoclonal antibody to the insulin-like growth factor I receptor blocks liganddependent signaling and inhibits human tumor growth in vivo. Cancer Res 2003, 63:8912-8921.

16. Wu JD, Odman A, Higgins LM, Haugk K, Vessella R, Ludwig DL, Plymate SR: In vivo effects of the human type i insulin-like growth factor receptor antibody A12 on androgen-dependent and androgen-independent xenograft human prostate tumors. Clin Cancer Res 2005, 11:3065-3074.

17. Yokota T, Kawakami Y, Nagai Y, Ma JX, Tsai JY, Kincade PW, Sato S: Bone marrow lacks a transplantable progenitor for smooth muscle type alphaactin-expressing cells. Stem Cells 2006, 24:13-22.

18. Cerwenka A, Morgan TM, Harmsen AG, Dutton RW: Migration kinetics and final destination of type 1 and type 2 CD8 effector cells predict protection against pulmonary virus infection. J Exp Med 1999, 189:423-434.

19. Kaufman LJ, Brangwynne CP, Kasza KE, Filippidi E, Gordon VD, Deisboeck TS, Weitz DA: Glioma expansion in collagen I matrices: analyzing collagen concentration-dependent growth and motility patterns. Biophys J 2005, 89:635-650

20. Chetty A, Nielsen HC: Regulation of cell proliferation by insulin-like growth factor 1 in hyperoxia-exposed neonatal rat lung. Mol Genet Metab 2002, 75:265-275.

21. Izumi K, Kurosaka D, Iwata T, Oguchi Y, Tanaka Y, Mashima Y, Tsubota K: Involvement of insulin-like growth factor-I and insulin-like growth factor binding protein-3 in corneal fibroblasts during corneal wound healing. Invest Ophthalmol Vis Sci 2006, 47:591-598.

22. Zhang S, Smartt H, Holgate ST, Roche WR: Growth factors secreted by bronchial epithelial cells control myofibroblast proliferation: an in vitro co-culture model of airway remodeling in asthma. Lab Invest 1999, 79:395-405.

23. Darby I, Skalli O, Gabbiani G: Alpha-smooth muscle actin is transiently expressed by myofibroblasts during experimental wound healing. Lab Invest 1990, 63:21-29.

24. Dugina V, Fontao L, Chaponnier C, Vasiliev J, Gabbiani G: Focal adhesion features during myofibroblastic differentiation are controlled by intracellular and extracellular factors. J Cell Sci 2001, 114:3285-3296.

25. Serini G, Gabbiani G: Mechanisms of myofibroblast activity and phenotypic modulation. Exp Cell Res 1999, 250:273-283.

26. Hinz B: Formation and function of the myofibroblast during tissue repair. $J$ Invest Dermatol 2007, 127:526-537.

27. Hinz B, Celetta G, Tomasek JJ, Gabbiani G, Chaponnier C: Alpha-smooth muscle actin expression upregulates fibroblast contractile activity. Mol Biol Cell 2001, 12:2730-2741.

28. Carter R, Jain K, Sykes V, Lanning D: Differential expression of procollagen genes between mid- and late-gestational fetal fibroblasts. J Surg Res 2009, 156:90-94.

29. Goldberg SR, Quirk GL, Sykes WW, McKinstry RP, Kordula T, Lanning DA: Differential use of Erk $1 / 2$ and transforming growth factor beta pathways by mid- and late-gestational murine fibroblasts. J Pediatr Surg 2008, 43:971-976

30. Nonaka M, Pawankar R, Fukumoto A, Yagi T: Heterogeneous response of nasal and lung fibroblasts to transforming growth factor-beta 1. Clin Exp Allergy 2008, 38:812-821. 
31. Hinz B: Tissue stiffness, latent TGF-beta1 activation, and mechanical signal transduction: implications for the pathogenesis and treatment of fibrosis. Curr Rheumatol Rep 2009, 11:120-126.

32. Huang X, Yang N, Fiore VF, Barker TH, Sun Y, Morris SW, Ding Q, Thannickal VJ, Zhou Y: Matrix stiffness-induced myofibroblast differentiation is mediated by intrinsic mechanotransduction. Am J Respir Cell Mol Bio 2012, 47:340-348.

33. Shi $Y$, Dong $Y$, Duan $Y$, Jiang $X$, Chen $C$, Deng L: Substrate stiffness influences TGF-beta1-induced differentiation of bronchial fibroblasts into myofibroblasts in airway remodeling. Mol Med Rep 2013, 7:419-424.

34. Johnson LA, Rodansky ES, Sauder KL, Horowitz JC, Mih JD, Tschumperlin DJ, Higgins PD: Matrix stiffness corresponding to strictured bowel induces a fibrogenic response in human colonic fibroblasts. Inflamm Bowel Dis 2013, 19:891-903.

35. Liu F, Tschumperlin DJ: Micro-mechanical characterization of lung tissue using atomic force microscopy. J Vis Exp 2011, 54. doi:10.3791/2911.

36. Goffin JM, Pittet P, Csucs G, Lussi JW, Meister JJ, Hinz B: Focal adhesion size controls tension-dependent recruitment of alpha-smooth muscle actin to stress fibers. J Cell Biol 2006, 172:259-268.

37. Zhou Y, Huang X, Hecker L, Kurundkar D, Kurundkar A, Liu H, Jin TH, Desai L, Bernard K, Thannickal VJ: Inhibition of mechanosensitive signaling in myofibroblasts ameliorates experimental pulmonary fibrosis. J Clin Invest 2013, 123:1096-1108.

doi:10.1186/1465-9921-14-102

Cite this article as: Hung et al:: Role of IGF-1 pathway in lung fibroblast activation. Respiratory Research 2013 14:102.

\section{Submit your next manuscript to BioMed Central and take full advantage of:}

- Convenient online submission

- Thorough peer review

- No space constraints or color figure charges

- Immediate publication on acceptance

- Inclusion in PubMed, CAS, Scopus and Google Scholar

- Research which is freely available for redistribution 